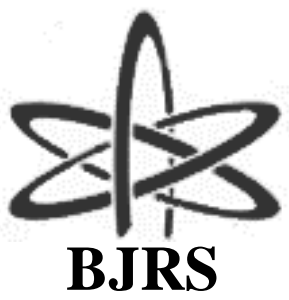
BRAZILIAN JOURNAL $\mathrm{OF}$ RADIATION SCIENCES
07-02B (2019) 01-14

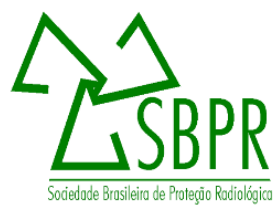

\title{
Study of volume fractions on biphasic stratified regime using gamma ray
}

\author{
W. L. Salgado $;$ L. E. B. Brandão ${ }^{\mathrm{a}}$ \\ ${ }^{a}$ Instituto de Engenharia Nuclear, 21.941-972, Cidade Universitária, Brasil \\ william.otero@hotmail.com
}

\begin{abstract}
In the oil industries, interconnected pipelines are used to carry large quantities of petroleum and its byproducts. This modal has an advantage because they are more economical, eliminate a need for stocks and, in addition, great safety in operation minimizing a possibility of loss or theft when transported another way. In many cases, especially in the petrochemical industry, the same pipeline is used to carry more than one type of product. They are called poliduct. In the operation of a poliduct there is a sequence of products to be transported and during the exchange of the product, there are still fractions of the previous product and this generates contaminations. It is therefore important to identify precisely this region in order to reduce the costs of reprocessing and treatment of discarded products. In this way, this work presents a methodology to evaluate the sensitivity of the gamma densitometry technique in a study of the calculation of volume fractions in biphasic systems, submitted to the stratified flow regime. Using computational simulations using the Monte Carlo Method with the MCNP-X code, measurement geometry was proposed that presented a higher sensitivity for the calculation of volume fractions. The relevant technical data to perform a simulation of the scintillator detectors were based on information obtained from the gammagraphy technique. The study had a theoretical validation through analytical equations, and the results show that it is possible to identify volume fractions equivalent to $3 \%$.
\end{abstract}

Keywords: volume fraction, gamma radiation, code MCNP-X

ISSN: 2319-0612

Accepted: 2019-02-19 


\section{INTRODUCTION}

The transportation of substantial quantities of products is being carried out by a system of interconnected pipes, such as oil and gas pipes, defining a transport pipeline of products for long distances. The oil pipes are the most efficient means in the distribution of petroleum and its derivatives, such as: gasoline, diesel, kerosene, etc. The pipelines are more economical to transport large volumes, especially due to their immense efficiency and reduced transportation costs, eliminate the need for stocks and present great safety in operation, minimizing the possibility of losses or thefts of products. The pipelines are commonly used within the industries has only one product, such as ducts that link offshore exploration platforms to the coast and the pipes that distribute water. The flow through the pipe is established by a conveyor element, essentially water or air; In the case of petroleum products (gasoline, diesel, ethanol, fuel oil, kerosene and naphtha), the pipelines always operate pressurized, establishing the displacement of the fluid. In many cases, especially in the petrochemical industry, the same principle of the transport line is used to carry more than one type of product (poliduct).

During the operation of a poliduct, it is necessary to decide which products should be moved; that is, how the products are sequenced pumped inside the poliduct. In this operation should consider the type of flow, availability of products and, in particular, physicochemical characteristics of the products involved. During the change of the pumped product, in a period, two distinct products are in direct contact with one another inside the duct, and the contaminations are inevitable. This process defines a region in the fluid called the interface. During the interface, following products should be directed to tanks for reprocessing, to be treated in a separation unit [1] causing large costs [2]. The losses caused by the interface are recognized as being more prominent for some product pairs, and it is impossible to put them in direct contact with the pipe during the operation on the network.

Although conventional sensors can be used to determine fluid densities, they are expensive because of the high installation and maintenance costs, as they need to be in physical contact with the fluid, which can be abrasive/corrosive, causing damage to the sensors that need to be changed periodicaly.

This situation shows the need to develop methodologies to accurately identify the interface region of fluids in order to enhance transport in poliducts and to reduce the costs of pumping, reprocessing and treatment of the discarded products. In order to avoid these difficulties, techniques using radiation sources have been applied for detailed "observation" of flow characteristics, especially the dis- 
tribution, the density and the concentration of the liquid phases and measure both the velocity of flow displacement to predict the volume fractions [3] and the fluid densities [4-5].

The gamma densitometry has been applied to many areas, such as: chemical, mining and petroleum industry, flow measurements [7-9], and for monitoring oil and petroleum derivatives [10]. The Radiotracer Laboratory of the Institute of Nuclear Engineering has been developing methodologies for predicting the density of petroleum products and derivatives. The results showed that the proposed approach is satisfactorily applied to predict fluid volume fractions [6]. Nuclear techniques such as gamma densitometry can provide reliable measures of density fluctuation, improving accuracy and reducing costs.

The great advantage of this technique is the possibility of analyze in real time and without the need to interrupt the normal operation of the installation (non-invasive techniques) presenting reliability in the results. In addition, the high detection sensitivity of these nuclear devices allows the use of sealed radioactive sources in order to minimize the potential for radiological risk to workers' health. The proposed system does not cause damage or radiological contamination to the equipment being considered safe from the environmental point of view. These advantages make these techniques very important in the industry and for this reason, they have been investigated and improved by many researchers. In cases of transport lines, a detector records the transmitted gamma rays after passing through the fluid and the pipe walls being possible to determine the volume fractions of the fluids.

Thus, this work presents a methodology to evaluate the sensitivity of the gamma densitometry technique in the study of the calculation of fluid volume fractions in biphasic systems (water-oil) submitted to the stratified flow regime using the MCNPX code. The measurements reveal a different response from the interaction mechanisms of the radiation and the medium. The model developed in the code considers the main effects of the interaction of the radiation with the matter involved and the detectors. The study considered a geometry composed of a source of ${ }^{137} \mathrm{Cs}$, an acrylic pipe measuring $3.5 \mathrm{~cm}$ radius and two $\mathrm{NaI}(\mathrm{Tl})$ detectors, a position diametrically opposed to source of radiation to measure the beam transmitted and the other displaced 60 degrees from the first, to measure the scattered beam. 


\section{MATERIALS E METHODS}

The methodology consists in mathematical modeling the NaI(Tl) detectors by the MCNPX code. The relationship between resolution and energy was determined experimentally for the correction of the spectra obtained by simulation. The parameter used for experimental validation of the detector's model was the comparison of the experimental photopeak absolute efficiency curve with the simulated values. The spectrums of gamma sources were measured with the $\mathrm{NaI}(\mathrm{Tl})$ detector to well defined distance between source-detector, in the axial direction of the detector and, afterwards, the same geometry was reproduced in code [11].

\subsection{Mathematical Detector Model}

The detector's simulation was based on information obtained from the gammagraphy technique. Both dimensions and materials were used for the calculation with the MCNPX code [12-13]. The $\mathrm{NaI}(\mathrm{Tl})$ crystal density used was $3.667 \mathrm{~g} . \mathrm{cm}^{-3}$, the $\mathrm{MgO}$ powder density used was $2.0 \mathrm{~g} . \mathrm{cm}^{-3}$ [14] and the aluminum density was $2.7 \mathrm{~g} . \mathrm{cm}^{-3}$. The photomultiplier tube on the back of the crystal was treated as a $30 \mathrm{~mm}$ thick aluminum disk to account for backscattering [15]. The mathematical model considered $\mathrm{NaI}(\mathrm{Tl})$ scintillator detector with $31 \mathrm{~mm}$ (diameter) and $19 \mathrm{~mm}$ (thickness).

A special treatment provided in the MCNPX code: the Gaussian energy broadening (GEB) (card FTn) option has been used to fit the full energy peak shape of the pulse height distribution (PHD). The GEB is a special treatment provided in the MCNPX code (card FTn) option has been used to fit the full energy peak shape of the PHD, the GEB parameters have been set taking into account the resolution of the detector by the FWHM provided by radioactive sources [12-13, 16-19], for this it must to be inserted into the input file (INP), the mathematical model of the detector. A set of measurements were performed in order to determine the energy resolution curve of the real $\mathrm{NaI}(\mathrm{Tl})$ scintillator detector (crystal + housing + photomultiplier tube material equivalent) used for this work, and the curve's coefficients were introduced in the function provided by the MCNPX code that fits a Gaussian to the spectrum to make the proper corrections [12-13, 19].

The experimental absolute efficiency measurements for point sources were compared with the 
simulated results under the same conditions of the experimental setup to validate the simulation of the NaI(Tl) detector [20]. The PHD estimate (F8 tally), available in the code MCNPX, was used to obtain the deposited energy distribution per incident photon on the considered detector volume. The spectrometric system response on energy resolution and efficiency curves was determined by measuring standard sources $\left({ }^{241} \mathrm{Am},{ }^{60} \mathrm{Co},{ }^{152} \mathrm{Eu},{ }^{22} \mathrm{Na}\right.$ and $\left.{ }^{137} \mathrm{Cs}\right)$ [12-13]. In calculations, it has been considered the radiation background and the contributions due to interactions by Compton Effect.

\subsection{Volume Fractions}

The method is based of transmission of a gamma ray beam through a pipe containing multiphase fluid. A source of gamma radiation with sufficient energy to penetrate and be transmitted throughout the tube is positioned on one side of the pipe and the counts are recorded using a $\mathrm{NaI}(\mathrm{Tl})$ scintillation detector placed another side. The recording of the signal in the detector is proportional to the emission of the gamma rays from the radioactive source. High density fluids cause a decrease in the counting rate at the detector, while the lower density fluids result in an increase in the counting rate. Considering a simplified model of the stratified flow regime with the static liquid phases in a pipe with cylindrical cross-section area, knowing the dimensions and characteristics of the pipe and of the fluids, the intensity of the beam is given by Equation 1 .

$$
I(E)=I_{0} \cdot \exp \left(-\sum_{1}^{2} \alpha_{i} \mu_{i}(E) \cdot x\right)
$$

Equation 1

Where:

$I$ - transmitted intensity of gamma rays (photons. $\mathrm{cm}^{-2} \cdot \mathrm{s}^{-1}$ );

$I_{0}$ - initial incident intensity of gamma rays from the source (photons. $\mathrm{cm}^{-2} \cdot \mathrm{s}^{-1}$ );

$x$ - fluid thickness $(\mathrm{cm})$;

$E$ - volume fraction of fluid i;

E: incident radiation energy;

$\mu_{i}$ - total fluid attenuation coefficient $i$.

In the Compton scattering, the energy of the scattered photon is smaller than that of the incident photon, that is, the incident photon transfers a fraction of its energy to an electron from the weakly 
bound layers of an atom resulting in a photon of reduced energy, which is expressed by the Comton Equation. From the conservation equations of momentum and energy, it is possible to calculate the energy of the scattered photon through Equation 2.

$$
\mathbf{E}_{\mathbf{c}}^{\prime}=\frac{\mathbf{h v}}{1+\frac{\mathbf{h v}}{\mathbf{m}_{0} \mathbf{c}^{2}}(1-\cos \theta)}
$$

Equation 2

Where:

hv - incident photon energy $(\mathrm{keV})$;

$\theta$ - scattering angle relative initial direction;

$\mathrm{m}_{0} \mathrm{c}^{2}-$ resting energy of the electron $(511 \mathrm{keV})$.

When the energy of the incident photon is much greater than the energy of binding of the orbital electrons, Compton scattering overrides the other interactions (photoelectric and Rayleigh). In the case of photons with energies $(100 \mathrm{keV}-10 \mathrm{MeV})$ materials with low atomic number $(\mathrm{Z} \leq 40)$, Compton scattering is the largest mechanism of interaction.

The probability of Compton scattering is directly proportional to the photon energy and inversely to the atomic number. The number of scattered photons arriving at the detector can be obtained using Equation 3.

$$
S=\Phi_{0}(E) \cdot \varepsilon(E) \cdot t \cdot \exp \left(-\int_{l_{1}} \mu\left(E_{\gamma^{1}}\right) d l\right) \frac{d_{e} \sigma^{K N}}{d \Omega} \cdot \frac{\rho \cdot N_{a} \cdot Z}{A} \cdot \exp \left(-\int_{l_{2}} \mu\left(E_{\gamma^{2}}\right) d l\right) d V d \Omega
$$

Equation 3

Where:

$\Phi_{0}(\mathrm{E})$ - photon flux incident with energy (E);

$\varepsilon(E)$ - detector photopeak counting efficiency in scattered photon energy;

$\mathrm{t}$ - count time (s);

$\mu\left(E_{\gamma^{1,2}}\right)-$ linear attenuation coefficients for primary and scattered photons; 
$l_{1,2}$ - path lengths of the photons in the source sample to the scattering center and back to the detector;

$\mathrm{d}_{\mathrm{e}} \sigma^{\mathrm{KN}}-$ Klein-Nishina differential cross-section in energy (E) for a free electron, which is the scattering probability of a photon;

$\rho$ - density;

$\mathrm{N}_{\mathrm{a}}$ - avogadro number;

$\mathrm{Z}$ - atômic number;

A - mass number of material;

$\mathrm{d} \Omega$ - angle solid;

$\mathrm{dV}$ - differential volume considered for radiation and its interaction with matter.

In the case of a homogeneous sample and point source geometry, the terms of the integrals are constant. In addition, the Klein-Nishina differential cross-section will be constant for a fixed geometry and a known incident photon energy and flux. Therefore, the counting rate depends only on the density of the material examined and information obtained by this technique is strongly dependent on the density of the material, so that the variation of density the sample could be monitored.

For a measurement geometry with a pencil beam and perpendicular to the layers of fluids. The calculation of the fluid volume fraction (FVF) on biphasic systems under the stratified flow regime using the gamma attenuation technique can be obtained using the Equation 4 and Equation 5.

$$
\alpha_{1}=\frac{\ln \left(I / I_{2}\right)}{\ln \left(I_{1} / I_{2}\right)}
$$

Equation 4

$$
\alpha_{1}=1-\alpha_{2}
$$

Equation 5

Where:

$\alpha_{1,2}-$ volume fraction of fluid 1 or 2 ;

I - gamma Ray intensity recorded with pipe containing fluids 1 and 2;

$\mathrm{I}_{1,2}$ - intensity containing only fluid 1 or 2 . 


\subsection{Proposed Geometry Of The Detection System}

The detection geometry consists of a source of ${ }^{137} \mathrm{Cs}$ and two $\mathrm{NaI}(\mathrm{Tl})$ scintillation detectors, positioned around an acrylic tubing. The first detector (D1) is positioned diametrically opposite the source, in order to measure the transmitted beam, while the second (D2) has an angle of 60 degrees to the first, to measure the scattered beam; the pipe has internal diameter of $7 \mathrm{~cm}$, thickness wall of the $0.5 \mathrm{~cm}$, as shown in Figure 1. The interior of the pipe has been filled with different amounts of fluids (volume fractions) to investigate the attenuation of the gamma rays. The F8 tally was used to estimate the simulated PHD classified since 40 to $800 \mathrm{keV}$. This energy range was chosen because the spectra acquired considering the peak of $662 \mathrm{keV}$ of the ${ }^{137} \mathrm{Cs}$. The fluids used are water $\left(\mathrm{H}_{2} \mathrm{O}\right)$ and oil $\left(\mathrm{C}_{10} \mathrm{H}_{18} \mathrm{O}\right)$. The volume fractions were varied from $50 \%$ to $100 \%$ with 6 steps. It is important to mention that the total absorption peak and the whole spectra were used in the analytical equations in order to evaluate the relative errors in the real volume fractions and choose the best methodology.

Figure 1: Simulated geometry.

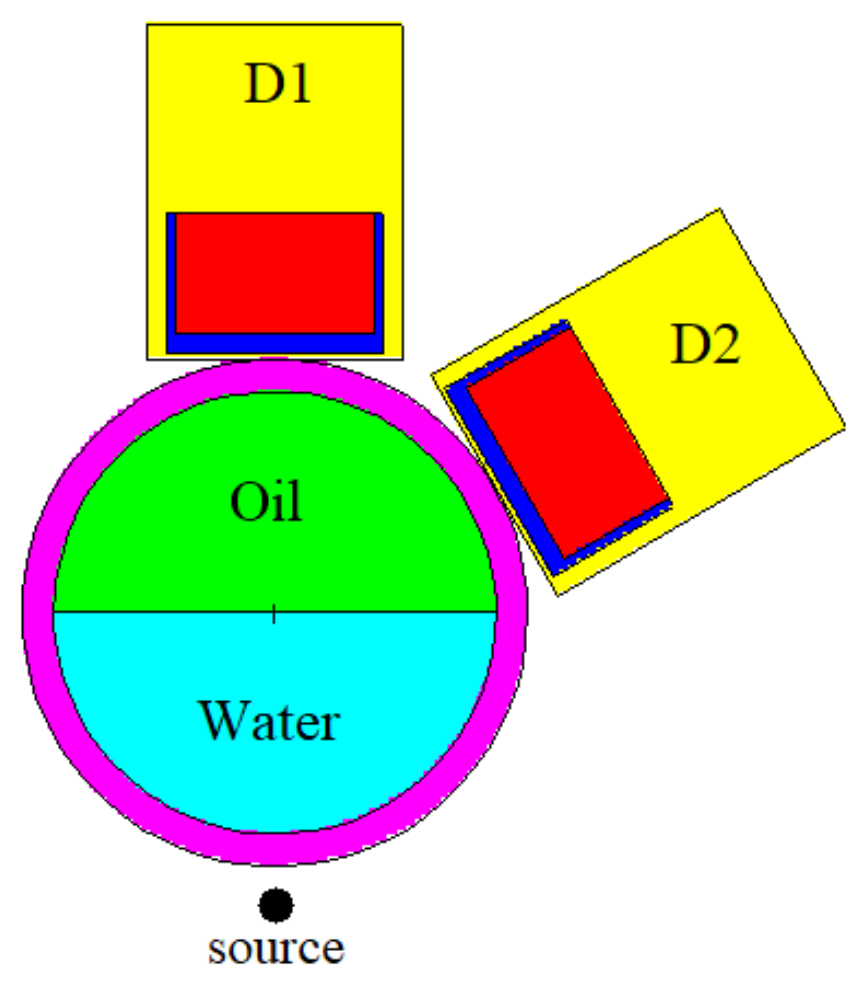




\section{RESULTS AND DISCUSSIONS}

\subsection{Detector Validation}

The detector's simulation was validated both qualitatively by the energy resolution curve and quantitatively by the photon detection efficiency. The gammagraphy technique showed to be an important tool to estimate, with some precision, the detector's dimensions used in this work. The aluminum disk at the base of the simulated detector takes into account the effect of all the materials of the photomultiplier tube located under the crystal. Figure 2 shows the schematic representation of the detector used for the simulation [12-13].

Figure 2: Schematic representation of the $\mathrm{NaI}(\mathrm{Tl})$ detector considered in simulation.

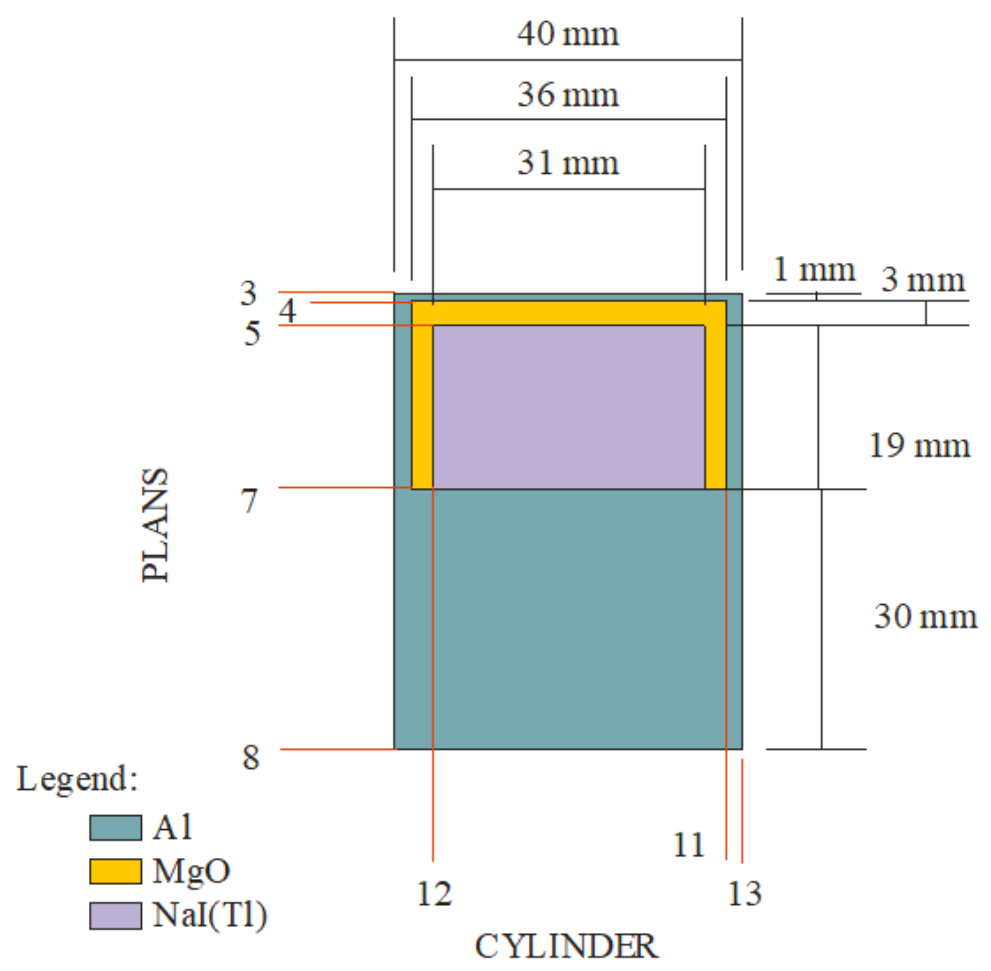

The Figure 3 shows the comparison between the experimental and simulated data by MCNP-X code for pulse height distributions to sources ${ }^{137} \mathrm{Cs}$. 
Figure 3: Comparison between experimental and simulated.

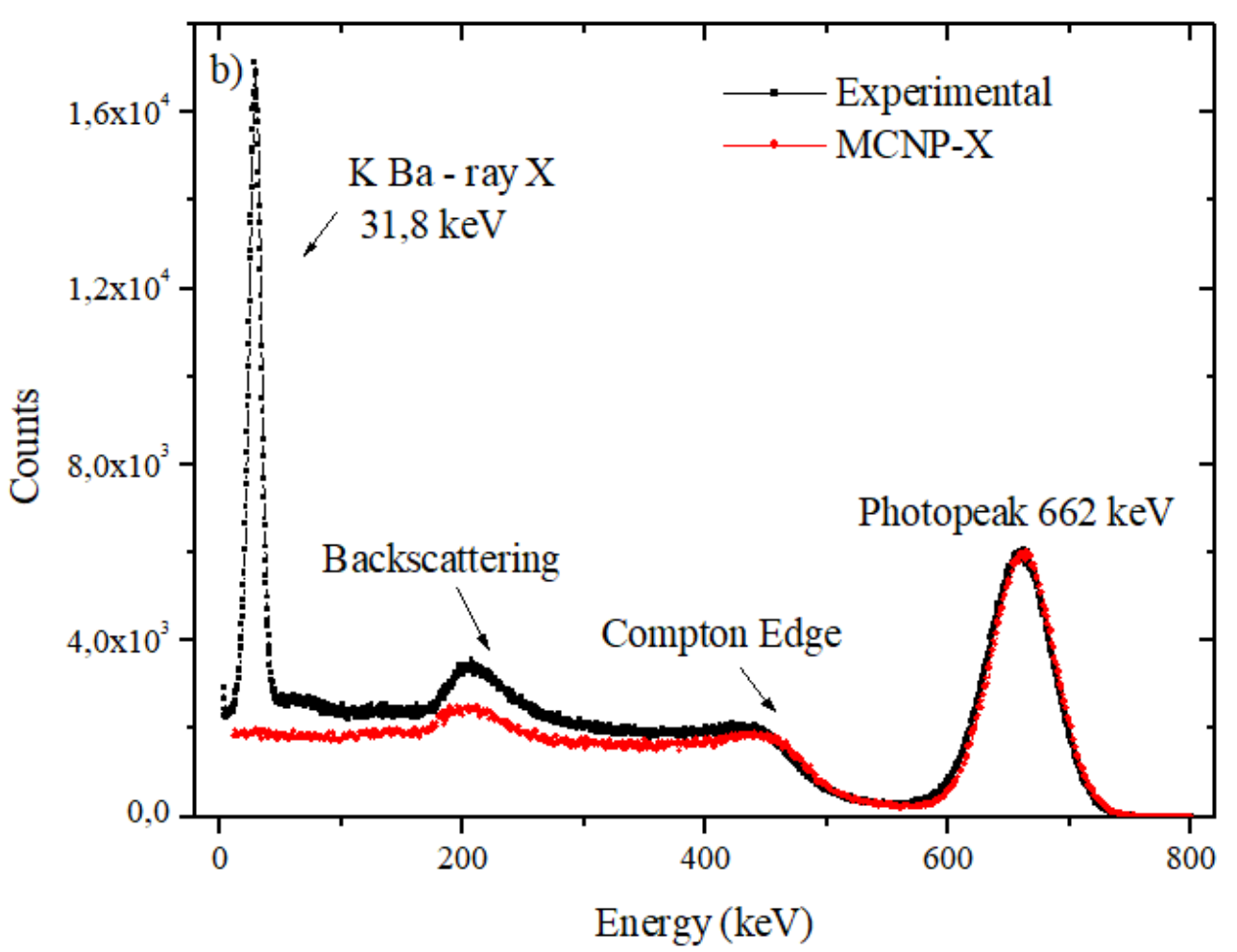

It is important to note that the spectrum shown in Fig. 3 had the subtraction of the background radiation. And it is possible to observe an acceptable agreement on the photopeak, for ${ }^{137} \mathrm{Cs}$ source, but in the Compton Effect region below $400 \mathrm{keV}$, all calculated values are lower than the experimental data due to the photons dispersed in the shield, the carrier and the materials around the measurement system, which were not considered in the simulation.

All simulated spectra were counted considering that the source activity represents the history number (NPS) used in the simulation in one second to obtain the counts recorded in the detector. The Xray peak was not simulated, so it does not appear in the spectrum obtained by the MCNP-X code.

\subsection{Volume Fraction Results}

For the study of the region of the spectrum to be analyzed by each method were simulated cases with only one fluid (Oil) with different void fractions equal to $0 \%, 25 \%, 50 \%, 75 \%$ and $100 \%$, the spectra obtained can be shown in Figure 4. 
Figure 4: Spectra obtained considering the volume void fractions: a) transmitted b) scattering.
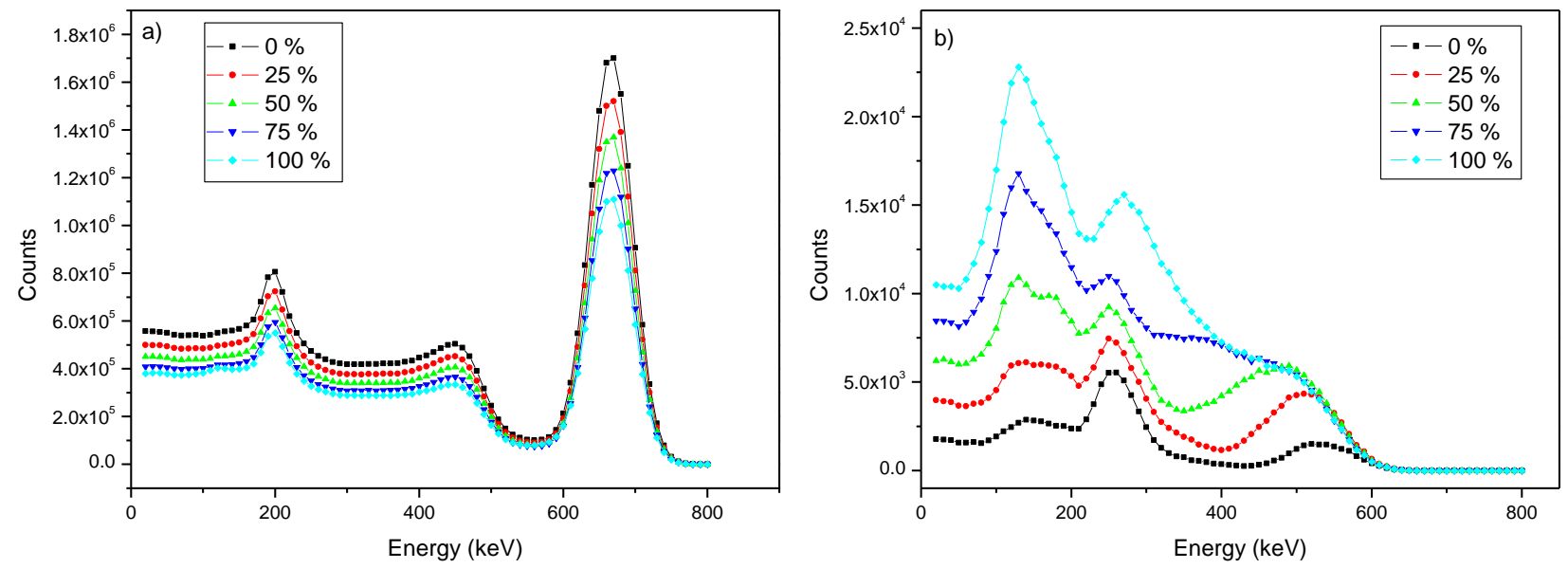

The calculation of the volume fraction using the measurements by transmission was performed by means of the counts referring to the photopeak obtained by the detector 1 , since it is the region of total absorption of energy being well characterized, as can be seen in Figure $4 a$. While for the scattering measurements obtained by detector 2 some regions of the spectrum were chosen since it is not possible to identify well defined peaks considering all the volume fractions studied, as can be seen in Figure 4b. The first chosen peak (peak CC) in $132 \mathrm{keV}$ and the all spectrum were regions that presented smaller errors in the calculation of volume fractions. For high vacuum fractions, there is a worsening of high energy peaks and an improvement in low energy peaks. For fractions of empty fractions, the opposite occurs. This makes it difficult to choose a single region for establishing the methodology.

The results for the volume fractions were obtained by means of Equation 4 and Equation 5, these results can be visualized in Table 1 .

Table 1: Comparison of the results obtained using analytical solution and the code MCNPX.

\begin{tabular}{cccc}
\hline \multicolumn{3}{c}{ Water Volume Fraction (\%) } \\
\hline \multirow{2}{*}{ Analitical Equation } & \multicolumn{3}{c}{ MCNPX Code } \\
\cline { 2 - 4 } & \multirow{2}{*}{ Detector $\mathbf{1}$} & \multicolumn{2}{c}{ Detector 2 } \\
\cline { 2 - 4 } & & All Spectrum & Peak CC \\
\hline 50 & 53.26 & 52.94 & 44.70 \\
\hline 59.06 & 60.66 & 60.83 & 53.54 \\
\hline 67.94 & 67.70 & 67.37 & 61.44 \\
\hline
\end{tabular}




\begin{tabular}{llll}
\hline 76.42 & 74.79 & 73.80 & 69.11 \\
\hline 84.29 & 81.65 & 80.14 & 76.26 \\
\hline 91.24 & 88.10 & 87.16 & 84.74 \\
\hline 96.83 & 94.12 & 94.14 & 92.31 \\
\hline
\end{tabular}

The relative errors for each of the performed procedures are shown in Figure 5. For the transmission spectrum and the all scattered spectrum, the maximum errors are around 5\%, while for the first peak (Peak CC) of the scattered spectrum, the errors reach values next to $10 \%$.

Figure 5: Relative errors in the calculation of volume fractions.

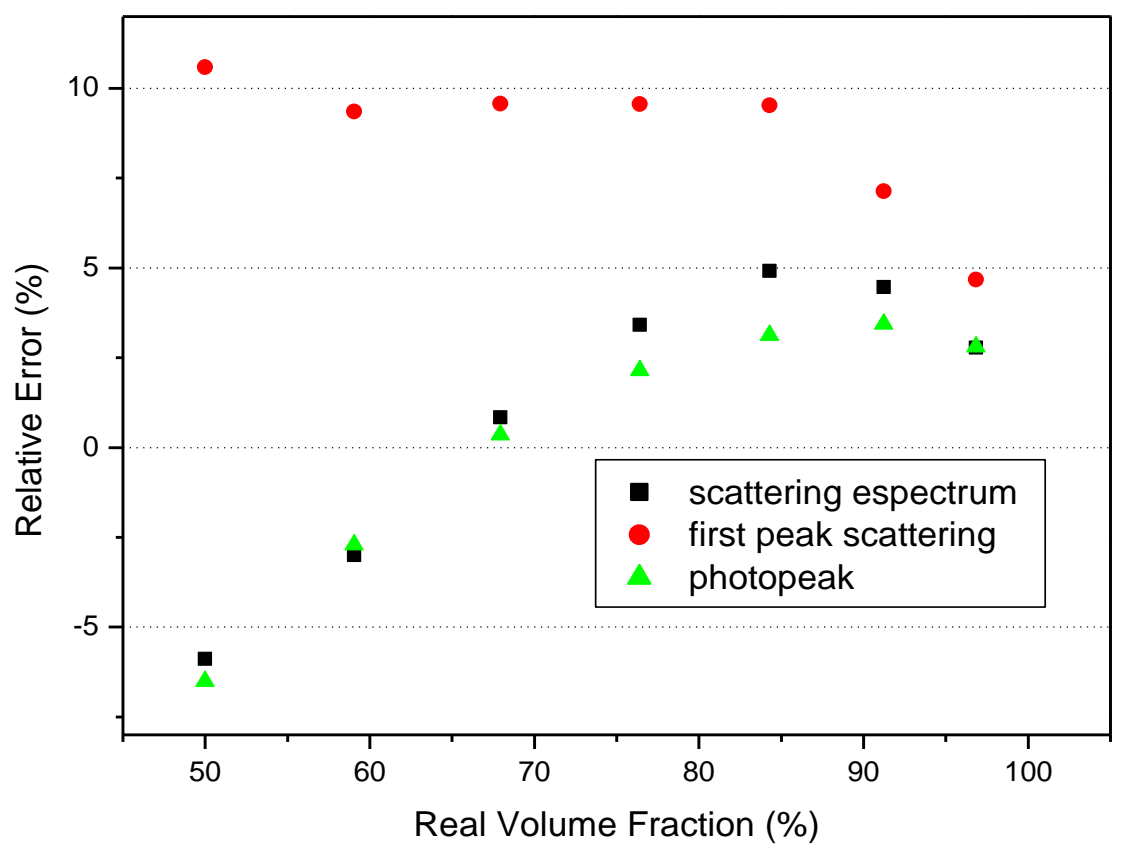

\section{CONCLUSIONS}

The measurement geometry developed in the MCNPX code together with the experimentally validated $\mathrm{NaI}(\mathrm{Tl})$ detector allowed us to give reliability to the methodology of calculation of volume fractions in biphasic (water-oil) systems in the stratified flow regime.

The results for the methodology of the transmission through the photopeak and the entire scattering spectrum indicate that it is possible to use both methodologies to calculate volume fractions of up to $3 \%$ with relative error around $\pm 5 \%$. However, the scattering methodology using the best peak region (peak CC) obtained a relative error of around 10\%. It is important to highlight that the experimental validation of the spectra for the scattering is still in the study phase. 
Studies using the methodologies together for an improvement in the sensitivity of the calculation of the volume fractions are in progress. In future works, these methodologies will be experimentally tested.

\section{REFERENCES}

1. Jones, W.M.C., Paddock, K.F., Transport by pipeline. In: Hobson, G.D., editor, Modern petroleum technology. Wiley, (1982).

2. Techo, R.; Holbrook, D.L., Computer scheduling the world's biggest product pipeline. Pipeline Gas Journal, 4:27, (1974).

3.AbouelwafaM.S.A. e Kendall E.J.M, “The measurement of component ratios in multiphase systems using gamma-ray attenuation", Journal of Physics E: Scientific Instruments,13, pp. 341-345 (1980).

4. Abdulrahman A.A., ShokirE.M., "Artificial neural networks modeling for hydrocarbon gas viscosity and density estimation". Journal of King Saud University - Engineering Sciences, 23, pp. 123-129, (2011).

5. C. M. Salgado, Cláudio M. N. A. Pereira, Luis E. B. Brandão e W.L. Salgado. Salinity independent volume fraction prediction in annular and stratified (water-gas-oil) multiphase flows using artificial neural networks.Progress in Nuclear Energy 76 pp. 17-23 (2014).

6. Salgado C.M., BrandaoL.E.B., Conti C.C. e Salgado W.L., "Density prediction for petroleum and derivatives by gamma-ray attenuation and artificial neural networks", Applied Radiationand Isotopes, 116 (2016) pp. 143-149.

7. Mi, Y., Ishii, M. e Tsoukalas, L.H., "Vertical two-phase flow identification using advanced instrumentation and neural networks". Nuclear Engineering and Design, 184, pp.409-420 (1998).

8. Salgado C.M., Brandão L.E.B., Nascimento C.M.N.A., Schirru R., Ramos R. e Silva A.X., "Prediction of volume fractions in three-phase flows using nuclear technique and artificial neural network", Applied Radiation and Isotopes, 67, pp. 1812-1818 (2009).

9. Salgado C.M., Pereira C.M.N.A., Schirru R. e Brandão L.E.B., "Flow regime identification and volume fraction prediction in multiphase flows by means of gamma-ray attenuation and artificial neural networks". Progress in Nuclear Energy, Vol. 52(6), pp. 555-562 (2010).

10. Khorsandi M. e Feghhi S.A.H., "Design and construction of a prototype gamma-ray densitometer for petroleum products monitoring applications". Measurement, 44, pp. 1512-1515, (2011). 
11. Salgado W. L., Silva A. X. A. e Salgado C. M., "Photopeak Efficiency Responde Function of an Underwater Gamma-ray NaI(Tl) Detector using MCNP-X”In: International Nuclear Atlantic Conference, 2015, São Paulo. Meeting on Nuclear Applications (XII ENAN), 2015.

12. Berger, M.J., Seltzer, S.M., "Response functions for sodium iodide scintillation detectors". $\mathrm{Nu}$ clear Instruments and Methods 104, pp.317-332 (1972).

13. Salgado, C.M., Brandão, L.E.B., Pereira, C.M.N.A., Robson Ramos, Schirru, R. e Silva, A. X., "Validationof a NaI(Tl) detector'smodeldevelopedwith MCNP-X code". Progress in Nuclear Energy 59, pp.19-25 (2012).

14. Saito, K. e Moriuchi, S., "Monte Carlo Calculation of accurate response functions for a NaI(Tl) detector for gamma rays", Nuclear Instruments and Methods 185 pp.299-308 (1981).

15. Hu-Xia Shi, Bo-Xian Chen, Ti-Zhu Li e D.I. Yun. "Precise Monte Carlo simulation of gammaray response functions for an $\mathrm{NaI}(\mathrm{Tl})$ detector", Applied Radiation and Isotopes 57, pp. 517-524 (2002).

16. Orion, I., Wilopolski, L., "Limitations in the PHOTON Monte Carlo gamma transport code". Nuclear Instruments and Meth.in Physics Research A 480, pp.729-733 (2002).

17. D. B. Pelowitz, MCNP-X TM User's Manual, Version 2.5.0. LA-CP-05-0369, Los AlamosNationalLaboratory (2005).

18. J.C. Vitorelli, A.X. Silva, V.R. Crispim, E. S. da Fonseca, W.W. Pereira, "Monte Carlo simulation of response function for a $\mathrm{NaI}(\mathrm{Tl})$ detector for gamma-rays from $241 \mathrm{Am} / \mathrm{Be}$ source", Appl. Radiat. Isot.62, pp.619-622 (2005).

19.C. M.Salgado, L. E. B. Brandão, R. Schirru, C. M. N. A.Pereira, R.Ramos e A. X.Silva, "Modelagem de detector NaI(Tl) usando MCNP-X". XI Encontro de ModelagemComputacional, n 0189, Volta Redonda, RJ (2008).

20. I.O.B. Ewa, D. Bodizs, S.Z. Czifrus e Z.S. Molnar, "Monte Carlo determination of full energy peak efficiency for a HPGe detector", Nuclear Instruments and methods in Physics Research A 479, pp.618-630 (2001). 